\title{
Investigation and Technological Comparison of 4G and 5G Networks
}

\author{
Yunman Hao \\ University of Central Lancashire, Preston, UK \\ Email:2924527459@qq.com
}

How to cite this paper: Hao, Y.M. (2021) Investigation and Technological Comparison of $4 \mathrm{G}$ and $5 \mathrm{G}$ Networks. Journal of Computer and Communications, 9, 36-43. https://doi.org/10.4236/jcc.2021.91004

Received: December 10, 2020

Accepted: January 19, 2021

Published: January 22, 2021

Copyright (C 2021 by author(s) and Scientific Research Publishing Inc. This work is licensed under the Creative Commons Attribution International License (CC BY 4.0).

http://creativecommons.org/licenses/by/4.0/

\begin{abstract}
Mobile cellular data networks have allowed users to access the Internet whilst on the move. Many companies use this technology in their products. Examples of this would be Smart Meters in the home and Tesla cars having their "over the air updates". Both of these two companies use the 4G and 5G technology. So this report will include a technical overview of the technology and protocols (LTE Advanced) used in 4G and 5G networks and how they provide services to the user and how data is transferred within the networks. And there are lots of different parts about the network architecture between the $4 \mathrm{G}$ and $5 \mathrm{G}$ systems. This report will talk about some different parts between these two systems and some challenges in them.
\end{abstract}

\section{Keywords}

4G, 5G, Protocols, Networks, Mobile Communication

\section{Basic Knowledge of $4 \mathrm{G}$ and $5 \mathrm{G}$ Networks}

\subsection{Introduction of $4 \mathrm{G}$ Networks}

The fourth generation mobile communication system (4G for short) includes TD-LTE and FDD-LTE, which is also the combination of the third generation mobile communication system (3G) and WLAN. Here, we should note that LTE is only an upgraded version of $3 \mathrm{G}$ and in fact it has not yet reached the $4 \mathrm{G}$ standard. The upgraded LTE Advanced is the standard version of $4 \mathrm{G}$, which meets the definition of $4 \mathrm{G}$ by the International Telecommunication Union.

The advantage of $4 \mathrm{G}$ is that it can transmit data very quickly, with high quality video, audio and image. The transmission speed of $4 \mathrm{G}$ is very fast. By definition, we regard the communication technology that can achieve the data transmission speed above $100 \mathrm{Mbps}$ (which is about the download speed of $12.5 \mathrm{MB} / \mathrm{s}-18.75$ 
$\mathrm{MB} / \mathrm{s}$ ) as $4 \mathrm{G}$. This technology is about 20 times that of home broadband ADSL, and can meet the requirements of most users for wireless services. $4 \mathrm{G}$ provides great convenience for the masses in communication, entertainment and business. Compared with previous generations of communication technologies, $4 \mathrm{G}$ has incomparable advantages, mainly in that $4 \mathrm{G}$ can be deployed in places not covered by DSL and CATV modems, and then extended to the whole region. In fact: $4 \mathrm{G}$ is the same as LTE technology, and they are both evolved from the existing $3 \mathrm{G}$ wireless communication technology. In other words, $4 \mathrm{G}$ is an advanced form of $3 \mathrm{G}[1]$.

As the most important protocol of $4 \mathrm{G}$ communication technology, LTE Advanced has two different standards: TD-LTE and FDD-LTE. These two modes correspond to two different duplex modes: TDD and FDD. TDD is what we call time division duplex. It means that uplink and downlink are crossed in the same frequency band according to time allocation, which can make better use of spectrum resources and facilitate layout; in addition, FDD is called frequency division duplex, which means that uplink and downlink are carried out simultaneously in different frequency bands. The advantage of FDD mode lies in its stronger data transmission capability. In fact, 4G uses OFDM to modulate downlink, while SC-OFDM to modulate uplink is quite different from 3G [2].

\subsection{Introduction of $5 G$}

\subsubsection{Development and Formulation Organization of 5G Protocol}

The official name of 5G is IMT-2020, which is the fifth generation mobile communication standard formulated by the International Telecommunication Union. As for the formation of $5 \mathrm{G}$ protocol, there is a unified standard in the industry field, instead of the multi-standard parallel standard in previous times. It can be said that the formation of $5 \mathrm{G}$ is the result of joint efforts of many organizations, among which $3 \mathrm{GPP}$ is the most important organization in standardization work. As a matter of fact, the reason why 3GPP was originally established was to customize technical reports and specifications for the third generation mobile communication system, and then it gradually evolved into the leading institution for formulation. As a standard, Release specified by $3 \mathrm{GPP}$ can complete the specification of one version in 1 - 2 years on average, and the first standard version of $5 \mathrm{G}$ is called R15 [3].

\subsubsection{G Protocol Framework}

As the first $5 \mathrm{G}$ standard of $3 \mathrm{GPP}, \mathrm{R} 15$ is mainly used to study the Phase 1 phase of $5 \mathrm{G}$. At this stage, we mainly study the definition of NR (the new global $5 \mathrm{G}$ standard based on OFDM is also the foundation of the next generation cellular network technology) framework, and clarify the waveform used by NR, flexible duplex mode of channel coding and frame structure. Solutions such as uplink and downlink decoupling, CU-DU separation, NSA/SA networking, etc. are clearly defined in the architecture, and the service types of 5G uRLLC are clearly defined. In Phase 2 of R16 protocol, the new multiple access technology of NR 
will be studied, and the framework of NR protocol will be further improved on the basis of R15. In addition to optimizing uRLLC on the basis of R15, R16 will also play its unique competitiveness in other vertical industries. The following is a chart to introduce it (Figure 1).

$5 \mathrm{G}$ is committed to research in eMBB, mMTC, uRLLC to provide services for people. EMBB refers to enhanced mobile broadband, which can provide users with larger system capacity and faster wireless access rate, so that users can enjoy a better ultra-clear experience. MMTC refers to mass Internet of Things, which is mainly to prepare for the wide popularization of Internet of Things in the future. This research can be applied to intelligent logistics, smart cities and even people's daily fitness. URLLC refers to high reliability and low delay. This research has been used in industrial production precision control, UAV (Refers to drone driving technology) remote monitoring and emergency personnel tracking, and it is believed that it will have a great impact on human life in the future [4].

\section{Comparison of Network Institutions}

\subsection{G Network Architecture}

The architecture of $4 \mathrm{G}$ network system consists of three parts, namely EPC, eNB and UE. Among them, EPS is composed of EPC and radio access network E-UTRAN, which is often called 4G network system. The core network EPC is mainly responsible for verifying user identity, transmitting user data and managing user data. The radio part (E-UTRAN) of $4 \mathrm{G}$ system mainly includes some functions of eNB and NodeB and some functions of core network. The specific framework is shown in Figure 2.

Actually, 4G network architecture can be understood as an architecture composed of LTE, SAE and IMS. LTE is the long-term evolution of air interface, SAE

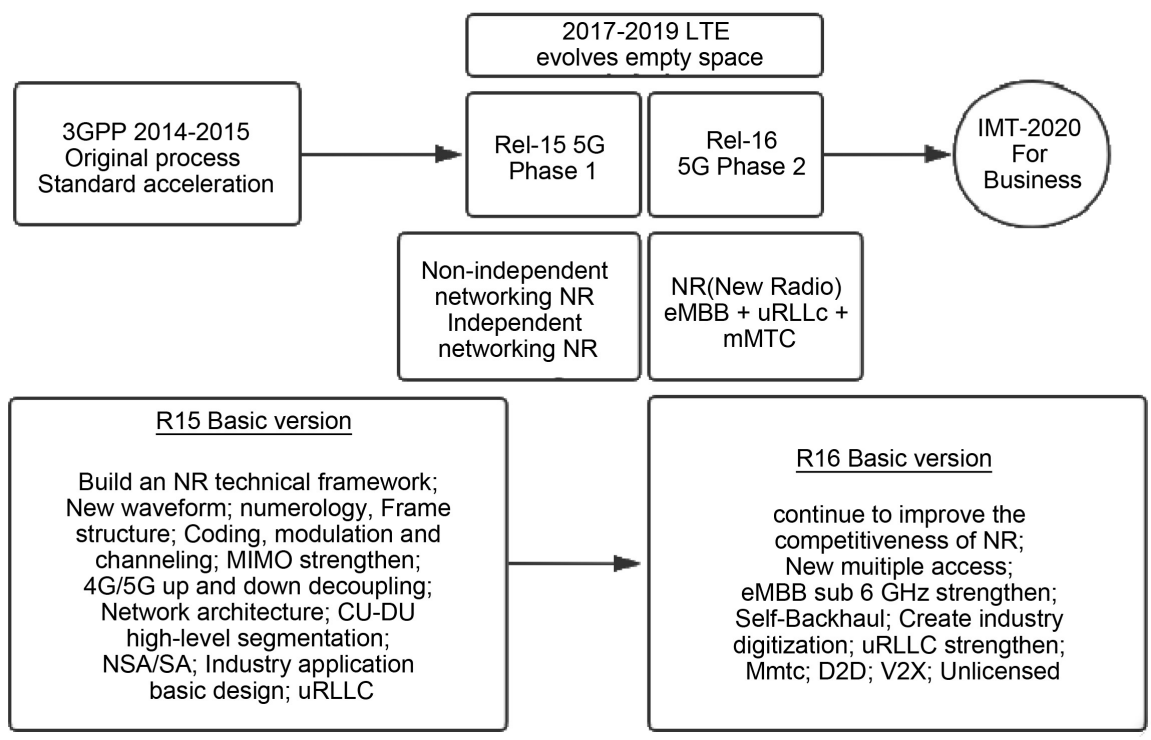

Figure 1. Protocol framework. 


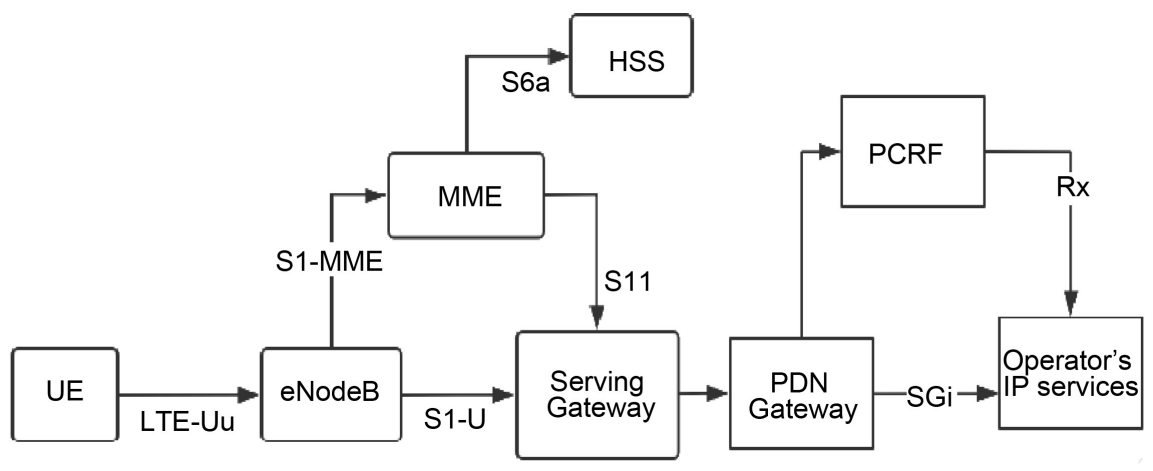

Figure 2. EPS network architecture.

is the evolution of system architecture, and IMS mainly solves the problem of carrying multimedia services such as voice to $4 \mathrm{G}$ network. The $4 \mathrm{G}$ basic architecture defined by GPP includes the following key points: First, MME is the control core of $4 \mathrm{G}$ network architecture, which is mainly responsible for handling control signaling such as user access control, service bearer control and handover control. Serving Gateway, as the anchor point when switching between local base stations, is mainly responsible for transmitting data information between base stations and public data gateways, and providing buffer for downlink data packets. Then PDN Gateway, as the anchor point of data bearer, can carry out packet forwarding, packet parsing, billing of basic related services, commercial QoS control, and so on. The full name of HSS is Home Subscriber Server. It is a database for storing contracts signed by users, and the stored information includes user security control information, user policy control information, user location information, user identification information and so on. Then, the full name of PCRF is Policy and Charging Rules Function. It mainly carries out policy control and charging control. It means that according to the contract selected and signed by the user, the user's service usage and charging strategy is determined and transmitted to the policy executor in the gateway, and finally the strategy is implemented.

\subsection{G Network Architecture}

The 5G network architecture adopts SBA architecture, which is called Service Based Architecture. In fact, $5 \mathrm{G}$ network is a user-centered intelligent elastic network, which splits the original whole into several individuals with independent functions, and these individuals play their own roles. Next, we analyze the $5 \mathrm{G}$ network in particular. First, we look at the unlimited network components of $5 \mathrm{G}$ network, which are $\mathrm{CU}, \mathrm{DU}$ and $\mathrm{AAU}$. CU is used to handle non-real-time services. DU is used to handle real-time business and physical requirements. The AAU is composed of RRU, passive wireless and a part of BBU physical layer. The architecture diagram of $5 \mathrm{G}$ unlimited network is shown in Figure 3.

\subsection{Comparison of 4G and 5G Network}

After analyzing and understanding $4 \mathrm{G}$ and 5G, we will compare these two 


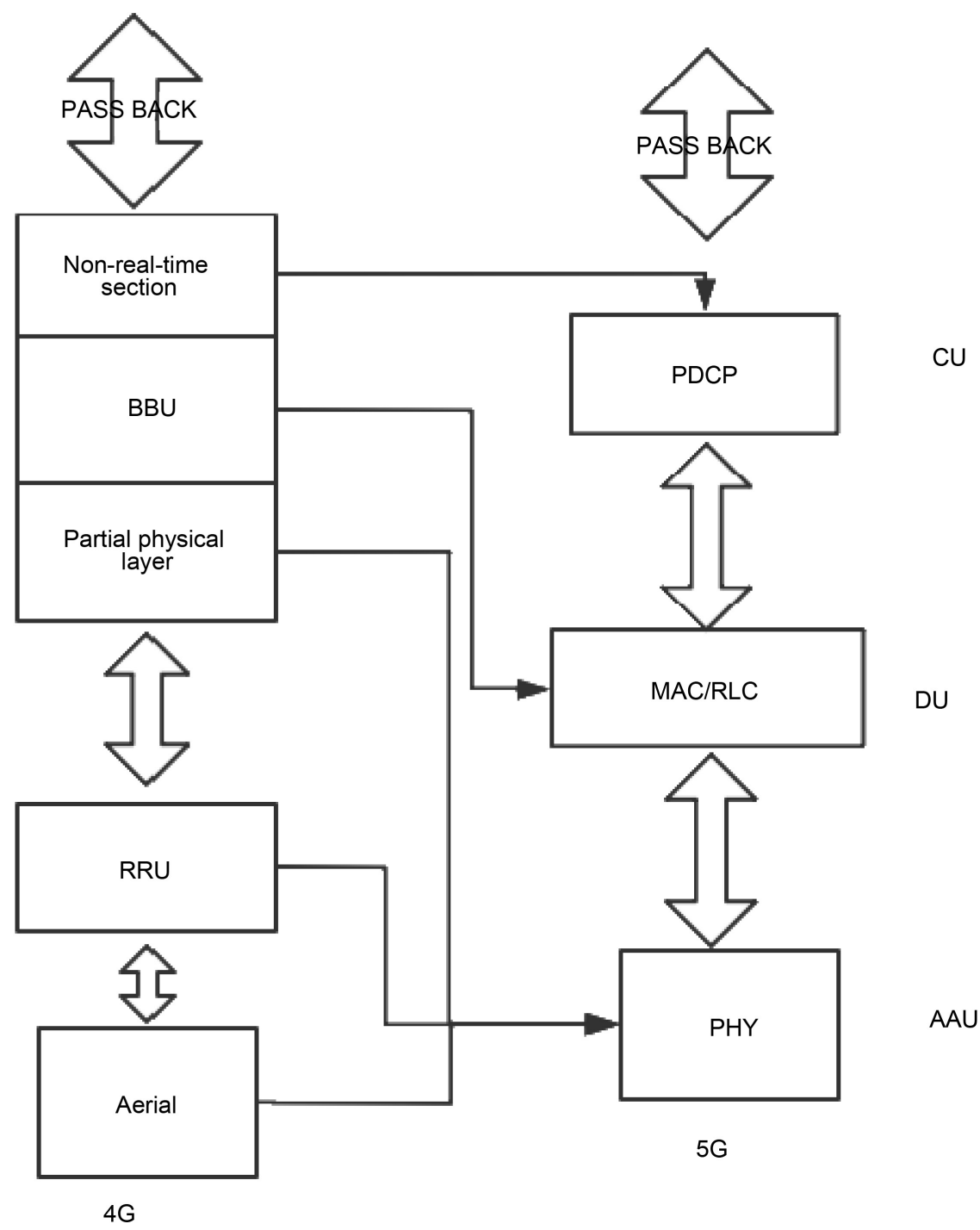

Figure 3. 5G unlimited network architecture.

network architectures. First of all, we know that the carrier of network communication is electromagnetic wave, but in fact, the frequency resources of electromagnetic wave are limited, and the higher the frequency, the faster the speed, and the more data that can be loaded, so the higher the frequency, the greater the broadband and the faster the speed. However, $4 \mathrm{G}$ uses low frequency band, so when many people use $4 \mathrm{G}$ network, there will be congestion, which will lead to lower rate. However, 5G does use high frequency band. In this case, even if many people use $5 \mathrm{G}$ network, there will be no rate reduction, and using high frequency can make better use of resources. In addition, the data download rate of $5 \mathrm{G}$ is as high as $10 \mathrm{Gbps}$, while that of $4 \mathrm{G}$ is about $100 \mathrm{Mbps}$, which is 100 times faster than that of $4 \mathrm{G}$. In addition, virtual industries such as VR, which have just become popular, are mostly based on $5 \mathrm{G}$ development. In addition, 5G network has the advantages of larger capacity, lower delay and stronger computing capability. At present, the most eye-catching industry is the communication 
industry, and the hottest industries in the communication industry are 4G and $5 \mathrm{G}$. At present, $5 \mathrm{G}$ is in the forming stage. We compare the performance of $4 \mathrm{G}$ and $5 \mathrm{G}$ in terms of transmission rate, frequency band, channel bandwidth and peak value [5]. The following is a performance comparison between $4 \mathrm{G}$ and $5 \mathrm{G}$ (Figure 4).

\section{Challenges of Implementing Mobile Communication Network}

\subsection{Challenges in Hardware Equipment}

Hardware equipment is the foundation of an experiment. Hardware equipment in mobile communication mainly includes baseband digital processing unit, frequency conversion and RF front-end devices. In order to obtain higher storage space and lower latency, 5G network uses a more advanced operation mode, which also brings greater challenges to its chip fabrication. For example, in order to meet the high frequency band of power amplifier above $4 \mathrm{GHz}$, the chip needs to use GaN material, which is very expensive and difficult to produce, so more efforts need to be made in hardware.

\begin{tabular}{|l|l|l|}
\hline & \multicolumn{1}{|c|}{$4 \mathrm{G}$} & \multicolumn{1}{c|}{$5 \mathrm{G}$} \\
\hline Time delay & $10 \mathrm{~ms}$ & smaller than 1 ms \\
\hline $\begin{array}{l}\text { Number of } \\
\text { mobile } \\
\text { links }\end{array}$ & eight billion & eleven billion \\
\hline $\begin{array}{l}\text { Channel } \\
\text { broadband }\end{array}$ & $\begin{array}{l}20 \mathrm{MHz} \\
200 \mathrm{MHz}\end{array}$ & $\begin{array}{l}100 \mathrm{MHz} \text { (lower than } 6 \mathrm{GHz} \text { ) } \\
400 \mathrm{MHz} \text { (larger than } 6 \mathrm{GHz} \text { ) }\end{array}$ \\
\hline $\begin{array}{l}\text { Frequency } \\
\text { band }\end{array}$ & $\begin{array}{l}600 \mathrm{MHz} \text { to } \\
5.925 \mathrm{GHz}\end{array}$ & $600 \mathrm{MHz}$ (millimeter wave) \\
\hline Date flow & $7.2 \mathrm{~Eb} / \mathrm{month}$ & $50 \mathrm{~Eb} / \mathrm{month}$ \\
\hline $\begin{array}{l}\text { Peak data } \\
\text { rate }\end{array}$ & $1 \mathrm{~Gb} / \mathrm{s}$ & $20 \mathrm{~Gb} / \mathrm{s}$ \\
\hline $\begin{array}{l}\text { Available } \\
\text { channel }\end{array}$ & $3 \mathrm{GHz}$ & $30 \mathrm{GHz}$ \\
\hline $\begin{array}{l}\text { Link } \\
\text { density }\end{array}$ & $\begin{array}{l}\text { One million } \\
\text { links/km*2 }\end{array}$ & One million links/km*2 \\
\hline $\begin{array}{l}\text { Uplink } \\
\text { waveform }\end{array}$ & Use SC-FDMA & Use CP-OFDM \\
\hline
\end{tabular}

Figure 4. Performance comparison. 


\subsection{Challenge of Flexibility of Network Architecture}

There are many kinds of services, and each service has different requirements for the network, so the diversity of services brings new challenges to the design and planning of 5G networks. It includes: function, resource utilization, architecture and so on. Secondly, the deployment, user category change and security isolation technology of $5 \mathrm{G}$ network need to be improved, which is one of the challenges to the flexibility of $5 \mathrm{G}$ network architecture. Compared with $4 \mathrm{G}$, the bandwidth of $5 \mathrm{G}$ network has increased dozens of times, which leads to the rapid increase of network speed demand. URLLC service requires ultra-low delay in milliseconds. This performance requires reasonable allocation of stations, optimization of routing and forwarding technology, and finally meets the flexibility requirements of $5 \mathrm{G}$ network.

\subsection{Challenges of Terminal Technology}

In order to meet the market demand, the terminal technology of $5 \mathrm{G}$ will be diversified. In fact, according to big data: "Most of the terminal products in the early $5 \mathrm{G}$ are mainly mobile phones in the eMBB scene, and the terminal planning of other scenes will gradually become clear with the maturity of standards and industries". From the perspective of current scientific and technological development, the increase in the number of antennas in the future will cause problems such as insufficient terminal space and reduced antenna efficiency. Therefore, researchers need to optimize the terminal design and hardware equipment, and then promote the development of the terminal industry chain [6].

\section{Conclusion}

This report is a technical overview of the technology and protocols used in $4 \mathrm{G}$ and $5 \mathrm{G}$ networks and how they provide services to the user and how data is transferred within the networks. Then, we make a comparison of the network architecture between the systems. Next, there is a description of the technical challenges in implementing mobile data communications and a table that compares technical properties of $4 \mathrm{G}$ and 5G. Finally, we make a conclusion on your findings and description of where you feel that the technology will progress. With the development of science and technology, 4G can no longer meet people's needs, while the research of $5 \mathrm{G}$ solves the problems of network delay and insufficient storage space. It can be seen that the performance of $5 \mathrm{G}$ is better than the previous network system. And I think that in the future, 5G will develop towards virtual industries like VR. Finally, it makes human life more convenient.

\section{Conflicts of Interest}

The author declares no conflicts of interest regarding the publication of this paper. 


\section{References}

[1] Zhou, S. (2011) Coverage and Networking Analysis of TD-LTE System. Papers 2 from: https://ieeexplore.ieee.org/document/6192901

https://doi.org/10.1049/cp.2011.0704

[2] Gibson, J.D. (2017) Mobile Communications Handbook (Electrical Engineering Handbook). 3rd Edition, CRC Press, Boca Raton, 56-83.

[3] Stuber, G.L. (2020) Principles of Mobile Communication. Springer International, Berlin, 24-47.

[4] Choi, Y.-I. and Park, N. (2017) Slice Architecture for 5G Core Network. Papers 10 from: https://ieeexplore.ieee.org/document/7993854 https://doi.org/10.1109/ICUFN.2017.7993854

[5] Ling, R. (2020) Mobile Communication. Wiley, Hoboken, 21-58.

[6] Liu, Y., Liu, H.M., Zhang, Y. and Guo, B. (2019) Go Deep into 5G Mobile Communication. China Machine Press, Beijing, Page 9. 